\title{
How harmless are E-cigarettes? Effects in the pulmonary system
}

Authors: Jack Bozier ${ }^{1,2 *}$, Razia Zakarya ${ }^{1,2}$, David Geoffrey Chapman ${ }^{3,4}$, Brian Gregory George Oliver ${ }^{1,2}$

1. Respiratory Cellular and Molecular Biology Group, Woolcock Institute of Medical Research, Sydney, Australia

2. Respiratory Cellular and Molecular Biology Group, School of Life Sciences University of Technology Sydney, Sydney, Australia

3. Translation Airways Group, School of Life Sciences, University of Technology Sydney, Sydney, Australia

4. Airway Physiology \& Imaging Group, Emphysema Centre, Woolcock Institute of Medical Research, Sydney, Australia

*Corresponding Author:

Name: Jack Bozier

Level 3, 431 Glebe point road, Glebe, Australia, 2037

Email: Jack.Bozier@sydney.edu.au

Phone: +61 0291140363 


\section{Abstract: \\ Purpose of review:}

Electronic cigarettes have quickly risen to be the leading alternative nicotine source to tobacco. Ecigarette use is hard to research and regulate due to the novelty and rapid evolution of the devices and E-liquids. Epidemiological data on long-term usage is currently lacking, but in smaller cohort studies we are starting to understand the usage patterns and demographics of users, which differ depending on where the study takes place and the regulatory environment. This review describes the current knowledge of the effects of E-cigarettes on the pulmonary system and knowledge of their usage patterns worldwide.

\section{Recent Findings:}

E-cigarette use is continuing to rise in young adults in USA and Canada, but not in UK. This suggests that regulation is influencing uptake in young adults. If E-cigarettes are to be considered as a harm minimisation smoking cessation product, use in young never smokers must be factored into the risk assessment. A recent surge in cases of lung injury associated with vaping in America has resulted in the definition of Vaping Associated Pulmonary Injury (VAPI), although the exact cause remains unknown.

\section{Summary:}

It is our opinion that E-cigarettes can no longer be defined as harmless. Further studies are needed to determine the risks for all populations as it is evident that a large proportion of E-cigarette users are never smokers, meaning they can't only be considered from a harm reduction perspective.

\section{Keywords:}

E-cigarettes, Smoking cessation, Inflammation, Respiratory disease 


\section{Introduction:}

The idea of an electronic nicotine delivery device as an alternative to tobacco was first conceived in 1963, but the first patented design came to market in 2003 [1]. Hon Lik originally designed the 'cigalike' devices as an alternative to combustible tobacco and their popularity has been rapidly increasing since their introduction to America and Europe in 2007 [2]. The devices have evolved to more advanced customisable products, but a shift towards Pod style devices in recent years suggests discretion and convenience is preferred by users. A good analogy to the different types of Ecigarette devices is home coffee machines. Some people prefer to roast, grind and brew their own coffee, whilst others opt for the convenience of prepacked coffee pods. The regulation surrounding E-cigarettes varies significantly throughout the world, although the development of devices and Eliquids by hundreds of different international manufacturers has left an insurmountable task for regulators to tackle [3]. It seems that it is universally agreed that they shouldn't be sold to minors, but beyond that opinions differ drastically on the harm versus benefit of E-cigarettes in different countries. In the United Kingdom a pro-E-cigarette position has been taken by Public health England, starting with the endorsement of Nutt et al. paper [4] proposing that E-cigarettes are $95 \%$ more healthy than tobacco cigarettes. This paper has been criticised for making conclusions without evidence to support their claims.

\section{E-cigarette uptake internationally}

In the USA and Canada a lack of regulation has contributed to an epidemic of E-cigarette use in young people. Recent data shows that E-cigarette use has significantly increased in young adults aged 16 to 19 between 2017 and 2018 [5]. E-cigarette use of 15 or more days in the last 30 days increased from $3.0 \%$ to $5.2 \%$ in USA and $2.1 \%$ to $3.6 \%$ in Canada. They also looked at changes in a cohort of 16 to 19 year olds from the UK, but no significant changes in prevalence of E-cigarette use 
were found. The United Kingdom was quicker to act on regulating E-cigarettes and USA waited on a congressional hearing about increasing prevalence of youth E-cigarette use, where it was revealed that Juul Labs was offering financial incentives to talk to students about the safety of e-cigarettes [6]. Australia is currently much further behind in the regulation of E-cigarettes, with regulation only allowing the sale of non-nicotine containing E-liquids in as nicotine is a registered poison with the Therapeutic Goods Administration.

Given the vast differences in international regulation of e-cigarettes it is important to evaluate recent evidence as to their effectiveness as a smoking cessation tool. A recent study by Hajek et al [7] compared the smoking cessation success of two cohorts, with one using nicotine replacement therapy (NRT) under the current therapeutic guidelines and the other using e-cigarettes as a replacement. They found that E-cigarettes were more effective as a cessation aid than NRT ( $18 \%$ vs 9.9\%). They also found that $80 \%$ of the E-cigarette cohort was still using their cessation product at a 52 week follow up, suggesting that they hadn't beaten their nicotine dependency. Conversely, a study by Gomajee et al [8] found that E-cigarette use in former smokers is associated with smoking relapse suggesting that E-cigarettes efficacy as a cessation aid could be more complicated than looking at short-term quit rates.

\section{E-cigarettes versus Tobacco}

The ongoing debate on whether E-cigarettes are more or less harmful than traditional tobacco cigarettes is delaying progress on regulation and distracting from the other major public health questions, particularly understanding the long-term population effects of E-cigarette use. It is clear that a lack of regulation is resulting in rapid uptake in younger non and never smokers, which may result in a rise in smoking rates among these populations $[9,10]$. One of the big unanswered questions is will the potential harm minimisation from E-cigarette use in current smokers outweigh the rate of smoking relapse and uptake of smoking by never smokers? The National Academies of 
Science, Engineering, and Medicine (NASEM) released a report in 2018 concluding that E-cigarette use results in reduced toxins, suggesting potential harm minimization [11].

Furthermore, the question on whether E-cigarettes are harmful as a stand-alone product, without comparison to cigarettes is still being debated. In human clinical trials and in real life pragmatic studies the different combinations of machine, machine operating parameters, liquids, and usage time initially led to a lot of confusion. In vivo and in vitro models have also been complicated by the lack of standard models, and the potential for models to be developed by those with commercial interests is worrying. However, even with this turbidity, it is hard to ignore the clear majority of studies concluding that E-cigarettes are cytotoxic, pro-inflammatory, genotoxic and effect respiratory function of users after a single session.

Higham et al [12] exposed Calu-3 cells and primary human bronchial epithelial cells in air liquid interface (ALI) culture to E-cigarette vapour extract resulting in an increase in proinflammatory cytokines IL-6 and CXCL8. The E-cigarette vapour extract was also cytotoxic to HBEC's. A similar study using Beas-2Bs epithelial cell line in ALI found that E-cigarette exposure was not cytotoxic, and relatively inert when looking at inflammatory mediator production [13]. Of the 10 inflammatory mediators measured only IL-6 was upregulated. Contradictory studies are regularly found due to the lack of a standardised E-cigarette exposure model. The study by Anthérieu et al outlines in their methods that they bubble a defined amount of vapour, $16 \times 3 \mathrm{~s}$ puffs through cell culture media, but Higham et al state their dose as an OD value. Anthérieu et al also received funding for their study from Innova, a French E-cigarette company, which should be considered as a conflict of interest. Without standard exposure times, volumes of E-vapour, clarity on the devices and E-liquids used we move further away from an answer on the health effects of using E-cigarettes. Regular publication of unreproducible data further adds to the confusion and delays action by regulatory bodies.

\section{Immunological impact of E-cigarettes}


Although the examples above point out some flaws in current E-cigarette research, it is evident that E-cigarettes have clear detrimental immunological effects in humans and on cells of the respiratory tract, cardiovascular system and immune system. Lugg et al [14] exposed primary alveolar macrophages (AM) to E-cigarette condensate finding upregulation of IL-6, TNF $\alpha$, CXCL8, MCP-1 and MMP-1. They also found that both nicotine and nicotine free vapour condensates were proapoptotic. Furthermore, addition of N-Acetyl Cystine (NAC) ameliorated the apoptosis suggesting ROS were responsible for the cytotoxicity. Ween et al [15] had similar findings in THP-1 macrophage cell line, with increased inflammatory markers after E-cigarette exposure and reduced phagocytotic ability. Post stimulation with E-cigarette vapour there was a reduction in expression of phagocytic surface receptors SR-A1 and TLR-2. Macrophages play a pivotal role in maintaining homeostasis within the lung, phagocytosing invading pathogens and apoptotic/necrotic host cells. The loss of macrophage function could result in increased infection and ineffective turnover and recycling of dead cells. It has also been shown that macrophages from e-cigarette exposed mice had significantly reduced ability to phagocytose bacteria than the macrophages of control mice [16], confirming that macrophage function is effected in the lung and in culture. This study also looked at viral infection in mice after E-cigarette exposure, with a significant decrease in viral clearance and an increase in morbidity and mortality. Furthermore, a study exposing mice to a range of Flavoured E-cigarettes both with and without nicotine looked at the effects on features of house dust mite-induced allergic [17]. All nicotine containing E-cigarettes suppressed airway inflammation but did not alter airway hyperresponsiveness or airway remodelling. Compared to room air, nicotine-free cinnacide flavoured E-cigarette exposure resulted in reduced total leukocytes and eosinophils, with an increase in airway hyperresponsiveness. Banana pudding E-cigarette exposure resulted in increased soluble lung collagen. These findings suggest that different E-liquid flavours may have a negative impact on users with allergic airways disease. 
Higham et al [18] exposed primary human neutrophils to E-cigarette vapour to determine activation and inflammatory response. There was an increase in activation markers CD11b and CD66b after Ecigarette exposure and increased secretion of MMP-9, Neutrophil Elastase and CXCL-8. These findings suggest that E-cigarette use would result in an increase in neutrophilic activation and recruitment. Dysregulated neutrophilic inflammation contributes to pathology in Chronic Obstructive Pulmonary Disease through increased matrix metalloproteinase (MMP) activity suggesting a plausible mechanistic link between e-cigarette use and long-term respiratory disease . Clapp et al [19] also found altered neutrophil function and cytokine production after E-cigarette exposure. There was a decrease in phagocytosis from several different E-liquid extract exposures, further supporting the increased risk of infection due to e-cigarette use. The impact of increased MMP activity could be explained in Ghosh et al study, where E-cigarette users, smokers and nonsmokers received bronchial brushings to determine any changes in bronchial epithelial protein expression [20]. They found 113 uniquely altered proteins in E-cigarette users, and 78 proteins altered in both E-cigarette users and smokers. Furthermore, E-cigarette users and smokers both experienced frequent coughing, poor tolerance for the bronchoscope and had erythematous airway mucosa, with more irritability and redness in the e-cigarette users.

\section{Pulmonary and Cardiovascular complications from E-cigarette use}

Mucocilliary dysfunction after E-cigarette exposure has been found in an ex vivo model of mucocilliary transport using Bullfrog Pallets [21]. There were similar findings in HBEC's and in vivo in a Sheep model [22]. Exposure to E-cigarette vapor reduced airway surface liquid hydration and increased mucus viscosity of HBECs in a nicotine-dependent manner. In both ex vivo and in vivo models E-cigarettes caused a reduction in tracheal mucous velocity (TMV). Cystic fibrosis transmembrane conductance regulator ion channel (CTFR) is also functionally altered after Ecigarette vapour exposure [23]. CTFR dysfunction is a proposed mechanism for mucus obstruction in chronic bronchitis and is also found in Cystic Fibrosis. These studies suggest that E-cigarettes could 
worsen symptoms and lung function for patients with lung diseases. There is currently no data on Ecigarette use in cystic fibrosis patients, but there has been a steady increase in use in patients with COPD or long-term smokers at risk of developing COPD [24]. The use of E-cigarettes in the at risk and COPD groups was associated with worse pulmonary related health outcomes such as increased prevalence of chronic bronchitis or a greater likelihood of COPD disease progression. Primary human airway smooth muscle cells from COPD patients had increased production of pro-inflammatory mediators compared to cells from healthy patients [25]. E-cigarettes could exacerbate the chronic inflammatory environment in the lungs of COPD patients and shouldn't be considered as a safe option for smoking cessation. A maternal E-cigarette exposure model using mice measured inflammation and DNA methylation in mothers exposed to E-cigarettes and their offspring who were exposed second-hand when feeding from the mother [26]. Mothers had increased TNF $\alpha$, IL-6, IL1 $\beta$ measured after weening of pups, whereas offspring had increased TNF $\alpha$ and suppressed IL1 $\beta$. They also found a global increase in methylation. Although there is current paucity of literature on intergenerational impacts of maternal e-cigarette use [27], in utero nicotine exposure has been shown to cause epigenetic aberrations and induce asthmatic pathology in offspring that persisted into the third generation [28]. This is most pertinent as smoking women have been shown to swap to e-cigarettes during pregnancy, believing it is a healthier alternative to smoking [29], therein propagating not yet identified health risks to further generations.

E-cigarettes have been shown to effect respiratory mechanical function of young healthy male study participants [30]. A cohort of $30 \mathrm{E}$-cigarette users performed a range of lung function tests and their results were compared against 30 healthy non-E-cigarette users. They found a pattern indicative of peripheral airway obstruction or small airways dysfunction, confirming that E-cigarette use had acute effects on lung function. Conversely another study [31] found that E-cigarette use had no effect on lung function in both asthma patients and healthy controls. In this study participants completed a 1 hour vaping session of a high-grade and contaminant-free mixture of propylene glycol and glycerol, with no flavourings or nicotine. This study isn't representative of normal E-cigarette 
use should be repeated to include an E-liquid containing nicotine to show any harm minimisation potential if used as a smoking cessation device.

There has been a recent surge in hospital admissions in the USA due to lung injury associated with ecigarette use. This has resulted in the definition of Vaping Associated Pulmonary Injury (VAPI) [32] which the USA Center for Disease Control and Prevention defines as e-cigarette use in the 90 days prior to symptoms, presence of pulmonary infiltrates on chest computed tomography imaging and no evidence of alternative diagnoses such as pulmonary infection or non-pulmonary disease. Four chest imaging patterns associated with VAPI have been identified; acute eosinophilic pneumonia, diffuse alveolar damage, organizing pneumonia, and lipoid pneumonia [33]. VAPI has been suggested to be characterised by lipid-laden alveolar macrophages, consistent with the recent finding that mice exposed to e-cigarettes had accumulation of lipids within alveolar macrophages and epithelial cells, altered surfactant homeostasis and impaired viral immunity. However, another study did not show lipid accumulation in macrophages in those with VAPI (ref). No common compound/ingredient has been identified, although the majority of patients reported use of Tetrahydrocannabinol or Cannabidiol (Layden NEJM 2019). However, given the large number of users of e-cigarette liquids containing cannabis, the relatively small number of cases, and the lack of regulation of e-cigarette liquid manufacturing in USA, there remains uncertainty as to the underlying cause of VAPI.

The health effects of E-cigarettes extend beyond the respiratory system, with growing concerns for cardiovascular health of E-cigarette users. The Health eHeart study [34] looked at how E-cigarette, cigarette and dual use influenced cardiopulmonary symptoms in patients. E-cigarette only use was associated with higher occurrence of chest pain, palpitations, coronary heart disease and arrhythmia. This study only has a low proportion of E-cigarette only users (1.4\%) and didn't identify whether any of these reported cardiovascular symptoms were from pre-existing conditions. Osei et al pooled data from the behavioural risk factor surveillance system, finding that E-cigarette users 
and concurrent smokers who use E-cigarettes were at greater risk of cardiovascular disease [35]. Further studies need to be done to identify how E-cigarettes affect the cardiovascular health of users vs non users. Lee et al [36] found that exposure to E-cigarette liquid caused endothelial cell dysfunction which is a precursor to myocardial infarction and other cardiovascular diseases. The exposure to un-vapourised E-liquid is not a physiologically relevant model as endothelial cells would only come into contact with the E-liquid in this form if it was ingested

\section{E-liquid flavours}

A regular focus point of in vitro studies is how flavour additives effect immunological response and cytotoxicity. It was last estimated in 2014 that there were more than 7500 different E-liquids available for purchase, this number is likely much larger today [37]. It is clear that the constituents of E-liquids affect the response from cells after exposure, including the flavour molecules in these liquids. Current regulation does not require manufacturers to test how harmful flavour molecules are on respiratory cells, or whether they are safe to inhale after vaporisation at high temperature. Krüsemann et al [37] proposed a new classification system for E-cigarette flavours with 13 main categories and 90 subcategories. They generated the categories from current E-liquid flavours names found in a literature search grouping them on common linking flavour groups they belonged to. A universal categorical system for E-cigarette flavours is a starting point for E-cigarette research with ingredients already known to have harmful effects, such as diacetyl [38], being banned from use. but much more needs to be done by the scientific community to set appropriate parameters on research expectations. A lack of universal in vitro and in vivo exposure models makes it difficult to compare two different studies. There is also very little transparency in the methodology used by researchers, further complicating the comparison of research to date.

One of the biggest dangers with E-cigarette use is its inherent novelty. Not only does it heighten its appeal, and make it hard to research and regulate; but also means there is a current dearth of epidemiological data on long-term usage consequences. Identifying any possible health risks is made 
more arduous by the comparison to cigarettes, which draws scientists and regulators into primarily assessing cigarette smoked induced changes and pathology as outcomes in studies. However, the basic constituents of E-cigarettes and cigarettes are radically different, and therefore E-cigarettes are likely to cause novel diseases. For example, inhalation of silicates in the form of asbestos causes radically different diseases to the inhalation of quartz [39]. Thereby we advocate for it to be of utmost importance that the scientific and medical communities are on high alert for novel pathological manifestations attributable to long-term E-cigarette use.

\section{Conclusion:}

It is our opinion that E-cigarettes can no longer be defined as harmless. Furthermore, the toxicity profile of e-cigarettes should not only be compared to tobacco cigarettes but considered in their own light i.e. as compared to never users. If e-cigarettes were prescription only smoking quit aids their utility and relative risk in the community would be very different to their use as recreational drug (nicotine) delivery system. When evaluating risks, differing points of view has led authors into presenting the data in ways to support their respective argument. We recommend further debate and studies to thoroughly discern the risks for all populations. In particular, studies focused on atrisk populations, such as teenagers, pregnant women, children, and people with pre-existing medical conditions need to be defined separately from those investigating risks associated with E-cigarette use as a quit aid or tobacco replacement product.

\section{Key Themes:}

- E-cigarettes can no longer be considered harmless, and should be considered in their own light without comparison to tobacco cigarettes.

- There is evidence for immunological, respiratory and cardiovascular complications from short or long-term E-cigarette use. 
- Regulations on E-cigarettes have an impact on the demographics that use them, hence further restrictions are needed to protect non-smokers and at risk populations.

\section{Acknowledgements}

We would like to thank the Woolcock Institute of Medical Research for providing the workspace to discuss this manuscript.

\section{Financial support and sponsorship}

No financial support was received for the writing of this manuscript.

\section{Conflicts of interest}

All authors have no conflicts of interest to declare. 


\section{References}

1. Wallace AM, Foronjy RE: Electronic cigarettes: not evidence-based cessation. Translational lung cancer research 2019, 8:S7-S10.

2. Jenssen BP, Boykan R: Electronic Cigarettes and Youth in the United States: A Call to Action (at the Local, National and Global Levels). Children (Basel, Switzerland) 2019, 6:30.

3. Zhu S-H, Sun JY, Bonnevie E, Cummins SE, Gamst A, Yin L, Lee M: Four hundred and sixty brands of e-cigarettes and counting: implications for product regulation. Tobacco control 2014, 23 Suppl 3:iii3-iii9.

4. Nutt DJ, Phillips LD, Balfour D, Curran HV, Dockrell M, Foulds J, Fagerstrom K, Letlape $\mathrm{K}$, Milton $\mathrm{A}$, Polosa $\mathrm{R}$, et al: Estimating the harms of nicotine-containing products using the MCDA approach. Eur Addict Res 2014, 20:218-225.

5. Hammond D, Reid JL, Rynard VL, Fong GT, Cummings KM, McNeill A, Hitchman S, Thrasher JF, Goniewicz ML, Bansal-Travers M, et al: Prevalence of vaping and smoking among adolescents in Canada, England, and the United States: repeat national cross sectional surveys. 2019, 365:12219.

6. HR293: Youth Vaping Prevention Act of 2019 116th Congress edition; 2019.

7. Hajek P, Phillips-Waller A, Przulj D, Pesola F, Myers Smith K, Bisal N, Li J, Parrott S, Sasieni $P$, Dawkins $L$, et al: A Randomized Trial of E-Cigarettes versus NicotineReplacement Therapy. 2019, 380:629-637.

8. Gomajee R, El-Khoury F, Goldberg M, Zins M, Lemogne C, Wiernik E, Lequy-Flahault E, Romanello L, Kousignian I, Melchior M: Association Between Electronic Cigarette Use and Smoking Reduction in FranceAssociation Between Electronic Cigarette Use and Smoking Reduction in FranceAssociation Between Electronic Cigarette Use and Smoking Reduction in France. JAMA Internal Medicine 2019.

9. Corey CG, Ambrose BK, Apelberg BJ, King BA: Flavored Tobacco Product Use Among Middle and High School Students--United States, 2014. MMWR Morb Mortal Wkly Rep 2015, 64:1066-1070.

10. Glantz SA, Bareham DW: E-Cigarettes: Use, Effects on Smoking, Risks, and Policy Implications. Annu Rev Public Health 2018, 39:215-235.

11. National Academies of Sciences E, Medicine, Health, Medicine D, Board on Population H, Public Health P, Committee on the Review of the Health Effects of Electronic Nicotine Delivery S: In Public Health Consequences of E-Cigarettes. Edited by Eaton DL, Kwan LY, Stratton K. Washington (DC): National Academies Press (US)

Copyright 2018 by the National Academy of Sciences. All rights reserved.; 2018

12. Higham A, Bostock D, Booth G, Dungwa JV, Singh D: The effect of electronic cigarette and tobacco smoke exposure on COPD bronchial epithelial cell inflammatory responses. Int J Chron Obstruct Pulmon Dis 2018, 13:989-1000.

13. Anthérieu S, Garat A, Beauval N, Soyez M, Allorge D, Garçon G, Lo-Guidice J-M: Comparison of cellular and transcriptomic effects between electronic cigarette vapor and cigarette smoke in human bronchial epithelial cells. Toxicology in Vitro 2017, 45:417-425.

14. Scott A, Lugg ST, Aldridge K, Lewis KE, Bowden A, Mahida RY, Grudzinska FS, Dosanjh $D$, Parekh $D$, Foronjy $R$, et al: Pro-inflammatory effects of e-cigarette vapour condensate on human alveolar macrophages. 2018, 73:1161-1169. 
15. Ween MP, Whittall JJ, Hamon R, Reynolds PN, Hodge SJ: Phagocytosis and Inflammation: Exploring the effects of the components of E-cigarette vapor on macrophages. Physiological reports 2017, 5:e13370.

16. Sussan TE, Gajghate S, Thimmulappa RK, Ma J, Kim J-H, Sudini K, Consolini N, Cormier $\mathrm{SA}$, Lomnicki $\mathrm{S}$, Hasan $\mathrm{F}$, et al: Exposure to electronic cigarettes impairs pulmonary anti-bacterial and anti-viral defenses in a mouse model. PloS one 2015, 10:e0116861-e0116861.

17. Chapman DG, Casey DT, Ather JL, Aliyeva M, Daphtary N, Lahue KG, van der Velden $\mathrm{JL}$, Janssen-Heininger YMW, Irvin CG: The Effect of Flavored E-cigarettes on Murine Allergic Airways Disease. Sci Rep 2019, 9:13671.

18. Higham A, Rattray NJW, Dewhurst JA, Trivedi DK, Fowler SJ, Goodacre R, Singh D: Electronic cigarette exposure triggers neutrophil inflammatory responses. Respiratory Research 2016, 17:56.

19. Clapp PW, Pawlak EA, Lackey JT, Keating JE, Reeber SL, Glish GL, Jaspers I: Flavored e-cigarette liquids and cinnamaldehyde impair respiratory innate immune cell function. American journal of physiology Lung cellular and molecular physiology 2017, 313:L278-L292.

20. Ghosh A, Coakley RC, Mascenik T, Rowell TR, Davis ES, Rogers K, Webster MJ, Dang $\mathrm{H}$, Herring LE, Sassano MF, et al: Chronic E-Cigarette Exposure Alters the Human Bronchial Epithelial Proteome. 2018, 198:67-76.

21. Palazzolo DL, Nelson JM, Ely EA, Crow AP, Distin J, Kunigelis SC: The Effects of Electronic Cigarette (ECIG)-Generated Aerosol and Conventional Cigarette Smoke on the Mucociliary Transport Velocity (MTV) Using the Bullfrog (R. catesbiana) Palate Paradigm. Frontiers in physiology 2017, 8:1023-1023.

22. Chung S, Baumlin N, Dennis JS, Moore R, Salathe SF, Whitney PL, Sabater J, Abraham WM, Kim MD, Salathe M: Electronic Cigarette Vapor with Nicotine Causes Airway Mucociliary Dysfunction Preferentially via TRPA1 Receptors. Am J Respir Crit Care Med 2019.

23. Lin VY, Fain MD, Jackson PL, Berryhill TF, Wilson LS, Mazur M, Barnes SJ, Blalock JE, Raju SV, Rowe SM: Vaporized E-Cigarette Liquids Induce Ion Transport Dysfunction in Airway Epithelia. Am J Respir Cell Mol Biol 2018.

24. Bowler RP, Hansel NN, Jacobson S, Graham Barr R, Make BJ, Han MK, O'Neal WK, Oelsner EC, Casaburi R, Barjaktarevic I, et al: Electronic Cigarette Use in US Adults at Risk for or with COPD: Analysis from Two Observational Cohorts. 2017, 32:13151322.

25. Bozier J, Rutting S, Xenaki D, Peters M, Adcock I, Oliver BG: Heightened response to e-cigarettes in COPD. 2019, 5:00192-02018.

26. Chen H, Li G, Chan YL, Chapman DG, Sukjamnong S, Nguyen T, Annissa T, McGrath KC, Sharma P, Oliver BG: Maternal E-Cigarette Exposure in Mice Alters DNA Methylation and Lung Cytokine Expression in Offspring. 2018, 58:366-377.

27. Zakarya R, Adcock I, Oliver BG: Epigenetic impacts of maternal tobacco and evapour exposure on the offspring lung. Clinical Epigenetics 2019, 11:32.

28. Rehan VK, Liu J, Sakurai R, Torday JS: Perinatal nicotine-induced transgenerational asthma. Am J Physiol Lung Cell Mol Physiol 2013, 305:L501-507.

29. Wagner NJ, Camerota M, Propper C: Prevalence and Perceptions of Electronic Cigarette Use during Pregnancy. Matern Child Health J 2017, 21:1655-1661. 
30. Meo SA, Ansary MA, Barayan FR, Almusallam AS, Almehaid AM, Alarifi NS, Alsohaibani TA, Zia I: Electronic Cigarettes: Impact on Lung Function and Fractional Exhaled Nitric Oxide Among Healthy Adults. 2019, 13:1557988318806073.

31. Boulay M-Ė, Henry C, Bossé Y, Boulet L-P, Morissette MC: Acute effects of nicotinefree and flavour-free electronic cigarette use on lung functions in healthy and asthmatic individuals. Respiratory Research 2017, 18:33.

32. Triantafyllou GA, Tiberio PJ, Zou RH, Lamberty PE, Lynch MJ, Kreit JW, Gladwin MT, Morris A, Chiarchiaro J: Vaping-Associated Acute Lung Injury: A Case Series. Am J Respir Crit Care Med 2019.

33. Henry TS, Kanne JP, Kligerman SJ: Imaging of Vaping-Associated Lung Disease. 2019.

34. Wang JB, Olgin JE, Nah G, Vittinghoff E, Cataldo JK, Pletcher MJ, Marcus GM: Cigarette and e-cigarette dual use and risk of cardiopulmonary symptoms in the Health eHeart Study. PLOS ONE 2018, 13:e0198681.

35. Osei AD, Mirbolouk M, Orimoloye OA, Dzaye O, Uddin SMI, Benjamin EJ, Hall ME, DeFilippis AP, Stokes A, Bhatnagar A, et al: Association Between E-Cigarette Use and Cardiovascular Disease Among Never and Current Combustible-Cigarette Smokers. Am J Med 2019.

36. Lee WH, Ong S-G, Zhou Y, Tian L, Bae HR, Baker N, Whitlatch A, Mohammadi L, Guo $\mathrm{H}$, Nadeau KC, et al: Modeling Cardiovascular Risks of E-Cigarettes With HumanInduced Pluripotent Stem Cell-Derived Endothelial Cells. 2019, 73:2722-2737.

37. Krüsemann EJZ, Boesveldt S, de Graaf K, Talhout R: An E-Liquid Flavor Wheel: A Shared Vocabulary Based on Systematically Reviewing E-Liquid Flavor Classifications in Literature. Nicotine \& Tobacco Research 2018.

38. Rooy FGBGJv, Rooyackers JM, Prokop M, Houba R, Smit LAM, Heederik DJJ: Bronchiolitis Obliterans Syndrome in Chemical Workers Producing Diacetyl for Food Flavorings. 2007, 176:498-504.

39. Mossman BT, Churg A: Mechanisms in the Pathogenesis of Asbestosis and Silicosis. 1998, 157:1666-1680. 